Recepción: 20 / 03 / 2019

Aceptación: 19 / 04 / 2019

Publicación: 05 / 06 / 2019

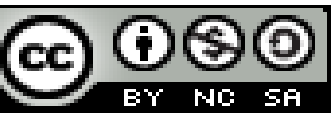

Ciencias de la salud

Artículo de revisión

\title{
Revisión teórica sobre las aseguradoras en Ecuador
}

\author{
Theoretical review on insurers in Ecuador
}

\section{Revisão teórica sobre seguradoras no Equador}

Vladimir A. Guerrero-Cortez ${ }^{\mathrm{I}}$ vladi.guerrero@hotmail.com

María E. Guevara-Toscano II maria.guevarat@ug.edu.ec

Marco A. Suriaga-Sánchez III marco_suriaga@hotmail.com

Correspondencia: vladi.guerrero@hotmail.com

I. Ingeniero Comercial; Magister en Tributación; Universidad de Guayaquil; Guayaquil, Ecuador.

II. Licenciada en Sistemas de Información; Magister en Administración de Empresas; Universidad de Guayaquil; Guayaquil, Ecuador.

III. Contador Público Autorizado; Magister en Tributación; Magister en Administración de Empresas Mención Negocios Internacionales; Universidad de Guayaquil; Guayaquil, Ecuador. 


\title{
Resumen
}

La necesidad humana de seguridad y la incertidumbre de ocurrencia de hechos inciertos, son las principales causas que contribuyen en la decisión de las personas y empresas de contratar una póliza de seguros, que en ciertos casos representa una inversión financiera. A nivel mundial la globalización y el desarrollo de las relaciones internacionales han ocasionado que el negocio asegurador se torne complejo, y para las compañías de seguros se ha convertido en un mercado más competitivo y exigente. Los seguros en el Ecuador han ido evolucionando y modernizándose, regulándose con nuevos requerimientos legales y normativos en busca de garantizar la solvencia de estas entidades. El presente artículo trata sobre: Funciones del Seguro; Importancia del Seguro; Seguros por la forma de prestación; Empresa de Seguros; Tipos de Seguros y Riesgos; y los seguros en el Ecuador. Se ha elaborado como un artículo de revisión, ahondando en un tema de mucho interés para quienes escriben, valorando la información disponible al respecto y construyendo un análisis con argumentos fundamentados en la literatura existente. El objetivo propuesto, es compilar suficiente información sobre el tema en estudio, utilizando para ello como metodología el análisis del discurso. Se ha querido exponer en el presente, los fundamentos formales que dan sustento a un trabajo de investigación en proceso. No se presenta como tal un cuerpo de conclusiones, se ha querido más que eso, compartir un conjunto de reflexiones que dan sustento a la revisión teórica realizada.

Palabras claves: Funciones del Seguro; Importancia del Seguro; Empresa de Seguros; Tipos de Seguros y Riesgos; Los seguros en el Ecuador

\begin{abstract}
The human need for security and the uncertainty of the occurrence of uncertain facts are the main causes that contribute to the decision of individuals and companies to take out an insurance policy, which in certain cases represents a financial investment. Globally, globalization and the development of international relations have caused the insurance business to become complex, and for insurance companies it has become a more competitive and demanding market. Insurance in Ecuador has been evolving and modernizing, regulating new legal and regulatory requirements in order to guarantee the solvency of these entities. This article deals with: Insurance Functions; Importance of Insurance; Insurance for the form of benefit; Insurance Company; Types of Insurance and Risks; and insurance
\end{abstract}


in Ecuador. It has been developed as a review article, delving into a topic of great interest to those who write, assessing the available information and constructing an analysis based on arguments based on existing literature. The proposed objective is to compile enough information about the subject under study, using discourse analysis as a methodology. It has been wanted to expose in the present, the formal foundations that give sustenance to a work of investigation in process. It is not presented as such a body of conclusions; it has been wanted more than that, to share a set of reflections that give sustenance to the theoretical revision made.

Keys words: Insurance Functions; Importance of Insurance; Insurance company; Types of Insurance and Risks; Insurance in Ecuador

\section{Resumo.}

A necessidade humana de segurança e a incerteza da ocorrência de fatos incertos são as principais causas que contribuem para a decisão de indivíduos e empresas de contratar uma apólice de seguro, que em certos casos representa um investimento financeiro. Globalmente, a globalização e o desenvolvimento das relações internacionais fizeram com que o negócio de seguros se tornasse complexo e, para as seguradoras, tornou-se um mercado mais competitivo e exigente. O seguro no Equador vem evoluindo e se modernizando, regulando novos requisitos legais e regulatórios para garantir a solvência dessas entidades. Este artigo lida com: Funções de Seguro; Importância do

Seguro; Seguro para a forma de benefício; Companhia de Seguros; Tipos de Seguros e Riscos; e seguros no Equador. Ele foi desenvolvido como um artigo de revisão, aprofundando um tópico de grande interesse para aqueles que escrevem, avaliando as informações disponíveis e construindo uma análise baseada em argumentos baseados na literatura existente. O objetivo proposto é compilar informações suficientes sobre o assunto em estudo, utilizando a análise do discurso como metodologia. Foi querido expor no presente, as fundações formais que dão sustento a um trabalho de investigação em processo. Não é apresentado como tal um corpo de conclusões, foi querido mais que isso, compartilhar um conjunto de reflexões que dão sustentação à revisão teórica feita.

Palavras chaves: Funções de seguros; Importância do Seguro; Companhia de Seguros; Tipos de Seguros e Riscos; Seguro no Equador

\section{Introducción.}


Según la Real academia de la lengua española, la palabra seguro proviene del latín "Securus" que significa libre y exento de todo peligro, daño o riesgo. Pero en el campo de estudio que estamos haciendo referencia nos remitiremos a la Legislación sobre contrato de seguros, vigente en el Ecuador donde manifiesta que: Seguro es un “...Contrato por el cual una persona natural o jurídica, se obliga a resarcir pérdidas o daños que ocurran en las cosas que corren un riesgo en mar, aire o tierra...” De acuerdo a nuestra legislación y en otros países, se entiende al seguro como un contrato mediante el cual una de las partes, el asegurador, se obliga, a cambio del pago de una prima, a indemnizar a la otra parte, dentro de los límites convenidos, de una pérdida o un daño producido por un acontecimiento incierto; o a pagar un capital o una renta, si ocurre la eventualidad prevista en el contrato, (Becerra 2013).

Para Picazo, (2015) la inseguridad que se vive en nuestros días no es nueva, pues a través del tiempo ha existido, podríamos decir desde que se genera el comercio como una actividad económica, siempre existió el riesgo de que las mercaderías no lleguen a su destino, ya sea porque los sistemas o medios de transporte no eran seguros, o porque los mercaderes podrían sufrir cualquier quebranto en sus salud, o que los productores no cumplieran con el despacho a tiempo de los solicitado, junto a esta necesidad nace la actividad comercial e intermediaria de la finanzas, con el fin de resarcir en parte o totalmente los daños que pudieran haber sufrido las personas y los bienes involucrados en un acto de comercio.

Los seguros están regulados por legislaciones en cada país. En el Ecuador, la norma aplicable es la “Ley General de Seguros” 1551, promulgada en el Registro Oficial № 547 del 21 de julio de 1965; y reformada el 25 de febrero de 1981 y por última vez el 12 de septiembre del 2014 donde se le atribuye a la Superintendencia de Compañías y Seguros su control y regulación. Es decir, en esta reforma se atribuye que: “...La constitución, organización, actividades, funcionamiento y extinción de las personas jurídicas y las operaciones y actividades de las personas naturales que integran el sistema de seguro privado; las cuales se someterán a las leyes de la República y a la vigilancia y control de la Superintendencia de compañías, valores y seguros, (Ley de seguros y reaseguros del Ecuador, 2014)

Los seguros no son un invento nuevo, sino que datan de muchos siglos atrás, cuando empezó la expansión del comercio a nivel mundial, la humanidad fue creciendo en cantidad, aumentando también las necesidades, por ello algunos pueblos como por ejemplo los fenicios, transportaban sus productos en barcos que navegaban distancias muy grandes para entregar las mercaderías hacia otras 
comarcas; la pérdida de las mercancías y de los recursos de quienes transportaban las mismas, originó la creación de seguros.

Técnicamente los seguros son un mecanismo para evitar que la pérdida de mercadería, infraestructura, activos o recursos, incluido la salud y la vida humana, puedan ocasionar crisis en las organizaciones y en los hogares.

El seguro como una herramienta de negocios, a nivel mundial, surge en el siglo XIV, desde ese entonces esta figura se fue legalizando con la aparición de la legislación en los diversos países del globo terrestre.

Alvear (2009), señala que el hombre desde su aparición tiene miedo a las pérdidas y a los gastos que se tiene a la falta de certidumbre y a la inseguridad personal, de sus bienes y de las personas que lo rodean. A mediados del siglo XIV surgió como un negocio vinculado al transporte marítimo de la floreciente industria italiana. De acuerdo a este autor, los seguros en el Ecuador, se originaron por la falta de seguridad que existía en el país, por el miedo a pérdidas materiales, de allí que se convirtió en un negocio que tenía vínculos con el transporte marítimo.

Para Durvan (2010), los Seguro son tan antiguos como la civilización misma; se encuentran antecedentes en las culturas griega y romana, y entre los aztecas; quienes concedían a los ancianos notables, algo similar a una pensión. Así, la historia del Seguro se remonta a las antiguas civilizaciones en donde se utilizaban prácticas que constituyeron los inicios de nuestro actual Sistema de Seguros. Probablemente las formas más antiguas de Seguros fueron iniciadas por los babilonios y los hindús. Estos primeros contratos eran conocidos bajo el nombre de Contratos a la Gruesa y se efectuaban, esencialmente, entre los banqueros y los propietarios de los barcos, a ello se mencionarían las etapas de evolución.

Se puede considerar al seguro como un producto básico, para el cual existe una demanda, dependiendo del precio. Algunas personas o empresas están dispuestas a ofrecer este producto y la cantidad ofrecida depende del precio. Bajo ciertas condiciones, existe un único precio en donde oferta y demanda se igualan, el precio de equilibrio. Sin embargo, el análisis va más allá el momento de introducir el riesgo y la proyección a futuro (Borch, 2004). 
El seguro actúa como una respuesta para mitigar la consecuencia de los riesgos, la manera más común para que las personas transfieran riesgos es comprando un seguro, en donde será la aseguradora quien se encargará de indemnizar todo o parte del prejuicio producido (Fundación Mapfre 2016).Desde el punto de vista de la solidaridad humana es la institución que garantiza un sustitutivo al afectado por un riesgo, mediante el reparto del daño entre un elevado número de personas amenazadas por el mismo peligro.

\section{Funciones del Seguro}

Blanco (2000) explica que, dentro de las funciones del seguro, éste promueve el ahorro ya que las reservas permiten realizar inversiones. "El seguro es un poderoso medio de captación y distribución del ahorro" (Blanco, 2000). En el sistema de seguro, las personas invierten hoy para cubrir sucesos en el futuro.

Es la misma visión de Arrow sobre el seguro: la gente transfiere su riesgo, pero a la vez está transfiriendo dinero y haciendo una inversión. También permite ampliar el rango de inversiones seguras y promueve el ahorro en personas de bajos recursos, tiene la tendencia de reducir distribuciones desiguales de riqueza (Arrow 1971)

Ya en 1601, Francis Bacon consideró que el seguro "es la estrella polar del comerciante que le induce a aventurar su capital" (Tortella, 2011). Actualmente, el seguro abarca muchos ramos y en cierta medida reduce el miedo de la gente a invertir, especialmente en actividades de emprendimiento o compras de activos que implican grandes sumas de dinero. Sobre todo, en caso de pequeños emprendedores ya que una pérdida es mucho más perjudicial cuando el patrimonio es menor.

Para Blanco (2000), la gente también se siente confiada cuando observa que las empresas tienen contratado seguros para proteger sus actividades, bienes y capital humano contra cualquier riesgo.

En otros casos, por ejemplo, las sociedades por acciones, comparten el riesgo y las posibilidades de beneficio entre todos los accionistas y así también se puede manejar un capital superior al que podrían manejar de manera individual. Y otra manera de invertir sin mucho riesgo es la diversificación en el mercado de capitales. Se adquieren diversas acciones y el comprador puede combinar la rentabilidad (Tortella, 2011). 
Otro beneficio que menciona Willet es la transferencia de riesgos. El seguro permite repartir riesgos que la sociedad no está dispuesta a correr, generalmente transferencias de riesgo de unidades económicas relativamente pequeñas a unidades muy grandes con capacidad de administración y solvencia (Tortella, 2011).

Arrow consideraba que hay una buena asignación del riesgo cuando éste se divide en varios mercados de seguros. Hay una asignación óptima bajo un sistema competitivo donde los valores se paguen en unidades monetarias (Arrow, 1964).

Habitualmente, la sociedad es adversa al riesgo, en términos de utilidad significa que tienen una utilidad marginal al ingreso decreciente. La transferencia permite a los dueños de negocios tener una mayor confianza en su producción porque les libera de la influencia de la incertidumbre y les permite concentrarse en otros aspectos del negocio o a la vez personales en caso que no sea un negocio. La ventaja para la sociedad de transferir riesgos se obtiene a través de la reducción de los costos que implica asumirlos ya que son asumidos por aquellos que tienen la capacidad de estimar riesgos, y a través del aumento en la eficiencia de aquellas personas que son altamente influenciables por la incertidumbre. El riesgo que asume una compañía de seguros es mucho menor que la suma de los riesgos del asegurado y a medida que la compañía se hace más grande, esta desproporción es mayor (Willet, 2001).

\section{Importancia del Seguro}

El hombre trata de prevenir posibles problemas que pueden generarse y de los cuales no hay certeza, por el contrario, existe incertidumbre. Para ello, un sector de seguros dinámico, organizado y controlado es muy importante para cubrir un mayor porcentaje de la población. Desde el punto de vista de la economía del bienestar, las pérdidas son riesgos, por los que las personas desean asegurarse. La no existencia de pólizas de seguro adecuadas y confiables implica una pérdida del bienestar. (Arrow 2003)

Willet (2001) menciona que cualquier cosa que disminuya el nivel de incertidumbre reduce el costo de riesgo para una sociedad. La combinación de riesgos y la consecuente reducción de incertidumbre es uno de los beneficios económicos del seguro e importantes para las personas. 
En referencia a la incertidumbre, Langlois (2010) señala que, debido a la existencia de incertidumbre, los mercados, cuando existen, pueden funcionar de manera imperfecta, entonces aquellas empresas que mejor dominen las condiciones de incertidumbre estarán en mejor disposición para poner en práctica sus estrategias

\section{Seguros por la forma de prestación}

Considerando la forma de prestación se distinguen los siguientes tipos de seguros:

- Seguros de indemnización

- Seguros por prestación de servicios

- Seguros de indemnización y servicios

Los seguros de indemnización se orientan al pago del importe pactado en el contrato de seguro ante la ocurrencia de riesgo, dentro de los seguros de vida en el Ecuador podemos encontrar; seguros por el fallecimiento del asegurado, vida entera o seguros temporales, beca estudiantil, seguros de ahorro o de supervivencia, enfermedades graves. (Albarrán y Del Pozo 2011).

Los seguros de prestación de servicios consisten en la realización de un servicio o asistencia al asegurado como ayuda para restablecer una condición desfavorable, por ejemplo, repatriación de restos mortales, asistencia en viajes, asistencia legal, asistencia dental, exequias, entre otros. (Albarrán y Del Pozo 2011) El diccionario de la Fundación Mapfre menciona: “en general, este concepto hace referencia a la prestación de una ayuda complementaria y organizada, dirigida a una determinada colectividad "; partiendo de esta definición podemos determinar a las asistencias como un sistema de prevención de riesgos que permiten a las compañías aseguradoras incurrir en menores costos que si lo asumieran por su propia cuenta.

Los prestadores de servicios permiten a las compañías de seguros diferenciar el producto de la competencia y brindar el soporte que garantice la satisfacción del asegurado a la hora de tangibilizar el servicio.

Una de las ventajas de las asistencias es que el asegurado accede a los servicios sin necesidad de desembolsar dinero en el momento de la prestación. Las formas de contratación varían de una 
compañía a otra, lo importante es que el cliente se asegure en elegir aquella asistencia que esté acorde a sus expectativas y se interese por condiciones básicas, por ejemplo: plazos de carencia, garantías, exclusiones, operatividad.

Los seguros de indemnización y de servicios son comercializados dentro de una misma póliza, de esta forma las aseguradoras aportan un valor agregado, ofreciendo a sus clientes adicionalmente a las coberturas indemnizatorias - sin costos visibles- asistencias para el hogar, familia y mantenimiento. (Albarrán y Del Pozo 2011).

Hoy en día muchas son las compañías aseguradoras ecuatorianas, que como parte de su estrategia de ventas añaden a las pólizas de seguros las llamadas asistencias gratuitas o prestaciones de servicios, de esta forma las empresas aseguradoras apuestan a la diversificación del negocio, mediante a la inclusión de estas nuevas y novedosas prestaciones.

\section{Empresa de Seguros}

Una Empresa de Seguros es una empresa de servicios cuya actividad económica consiste en ofrecer a personas u empresas la cobertura de posibles riesgos económicos. Los riesgos económicos son los riesgos asegurables, es decir cualquier bien personal o colectivo que sea tangible y cuantificable.

Como lo plantea (Medrano, 2008) la realización de estos riesgos se denomina siniestros. Los siniestros implican para las personas u empresas pérdidas no solo materiales si no también financieras. El rol de la empresa aseguradora es cubrir estas pérdidas. "Una compañía de seguros reduce pérdidas financieras provocadas por hechos aleatorios que impiden la realización de expectativas razonables". Cuando existen respuestas controladas a peligros que se pueden presentar en cualquier actividad representa un mecanismo de alta competitividad y desarrollo, facilitando el crecimiento del sector económico o crecimiento personal. La justificación económica de un sistema de seguros es que contribuye al bienestar general, mejorando las perspectivas de planes que podrían frustrarse por eventos negativos. Las organizaciones de seguros (aseguradoras) se establecieron para ayudar a disminuir las consecuencias financieras negativas de hechos inesperados. En este contexto las empresas de seguros son una garantía para el emprendimiento de nuevos proyectos y fundamentales para la toma de decisiones. 
Según Willet, (2001) una empresa de seguros debe realizar varios análisis para determinar el riesgo, la probabilidad de que suceda algún siniestro y la prima correspondiente. Sin embargo, este autor menciona que a pesar de que ninguna otra cosa es más esencial en los negocios que la habilidad de predecir sucesos futuros o deseos de los consumidores, es importante destacar que las pérdidas pueden resultar de la no ocurrencia de un evento anticipado, así como la ocurrencia de un evento no anticipado.

Las Empresas de Seguros deben ser capaces de utilizar adecuadamente la información disponible y con ella predecir con precisión mediante un análisis estadístico y de esta manera, agrupar el riesgo. El agrupamiento es una estrategia para diversificar el riesgo y consiste en que los individuos inviertan poco en muchos sucesos no relacionados entre sí. Este agrupamiento implica que a pesar de que las compañías de seguros protegen a los individuos frente al riesgo, las compañías no asuman grandes riesgos que no serán capaces de afrontar. Sin embargo, las compañías de seguros no pueden eliminar completamente el riesgo, "existe siempre reducto de riesgo que no se puede diversificar.

Las Empresas de Seguros como cualquier otra empresa maximizan su utilidad y existen debido a que existen ganancias derivadas del comercio del riesgo: transferir el riesgo de una persona más adversa a otra menos adversa que está dispuesta a asumirlo.

Las empresas de seguros se crean por su demanda. Los agentes económicos demandan seguros debido a la aversión frente al riesgo. Las personas están dispuestas a pagar un precio considerable a cambio de reducir su riesgo. Pagarán una prima que es una cantidad de dinero por una indemnización futura en caso de que se produjera una situación adversa, es el pago que realiza el asegurado a la compañía de seguros a cambio de la promesa de ésta de hacerse cargo de una serie de pagos en caso de que sucedan determinados siniestros.

Los asegurados pagarán una prima siempre y cuando ésta sea menor que la posible pérdida en caso que ocurra el siniestro, pero mientras más adversos al riesgo son, mayor es la primas que están dispuestos a pagar. Por el otro lado, una empresa aseguradora es neutral al riesgo de otros agentes económicos y como cualquier empresa, una aseguradora busca maximizar su utilidad, por lo tanto, su principal propósito es la rentabilidad financiera (Molina et al, 2010) 


\section{Tipos de Seguros y Riesgos}

Según Pinto (2001) el grupo de seguros está integrado por contratos de objeto semejante, los ramos son formas especiales de seguros y sus diferentes tipos son modalidades de seguros.

Se considera el ramo como el conjunto de modalidades de seguro relativas a riesgos de características o naturaleza semejantes. Este autor los clasifica en: seguro de cosas, seguros de derechos, seguros patrimoniales y seguros de personas.

Los seguros de cosas abarcan los ramos que aseguran un bien, como el seguro de incendio, robo, vehículos, de nave, etc. El grupo de seguros de derecho es aquel en el cual el interés del asegurado está en su derecho de exigir préstamos a terceros como el seguro de caución. Los seguros patrimoniales son aquellos que tratan de evitar el deterioro del seguro por un acto que lesiona a un tercero, es el caso del seguro de responsabilidad civil. El seguro de personas gira alrededor de la integridad personal del asegurador como el seguro de vida, accidentes, personales o de enfermedad.

Ahora bien, Ávila plantea (2008), que toda empresa enfrenta riesgos; los cuales define como riesgo interno, riesgo sectorial y riesgo de mercado.

- Riesgo Interno: Causado por debilidades de la empresa que hacen vulnerables a sus procesos por errores y falencias cometidas. Su control al ser propio de la empresa puede ser superado con decisiones correctas que eliminen las causas que lo provocan.

- Riesgo Sectorial: Causado por la competitividad propia de las empresas y personas naturales que participan en la actividad económica y atienden al mercado objetivo.

- Riesgo de Mercado: Causado por los factores externos no controlables que participan en el mercado y que establecen condiciones que inciden en el comportamiento del sector y de la empresa.

Las Empresas de Seguros para reducir el riesgo sectorial debido a la competencia, ajustan el nivel de primas en el mercado para captar más asegurados. Sin embargo, la prima se puede reducir solo a un cierto nivel para poder alcanzar ganancias. Y el riesgo más importante para una aseguradora es el 
interno debido a que depende de procesos complejos para poder asumir riesgos que no son propios y así mismo mientras mejores técnicas tengan menor competencia tendrán en el mercado.

Una empresa aseguradora se somete a mayores riesgos que otra empresa porque debe asumir riesgos propios y riesgos de otras personas u empresas, pero al mismo tiempo a mayor riesgo, existe mayor rentabilidad de acuerdo a la capacidad de la empresa de establecer las mejores técnicas y medidas preventivas y correctivas.

Según Willet (2001) cuando la empresa de seguros acumula riesgos iguales de muchos asegurados, la posibilidad de tener errores al predecir riesgos será menor, así el riesgo que una empresa de seguros lleva es mucho menor que la suma de los riesgos individuales de los asegurados. Además, mientras más seguros existan, se cumple el principio de compensación en donde la prima pagada por siniestros no ocurridos ayuda a cubrir los siniestros que sí sucedieron. Y mientras mayor número de pólizas emitidas, más grande es la empresa. Mientras más grande una empresa de seguros es, puede brindar más barato el servicio de seguros, a mayor riesgo, más barato el seguro. Esto debido a que el peligro agregado es mucho menor que la suma de los peligros individuales.

El mecanismo de una empresa aseguradora es expedir pólizas, las cuales son pagadas por el asegurado con la prima. Esta póliza promete pagar al beneficiario la pérdida financiera del siniestro ocurrido. Este sistema de seguros permite transferir riesgos de una persona o empresa que es el asegurado a una entidad que asume este riesgo y es la empresa aseguradora. Las empresas de seguros resuelven el problema del riesgo. Por lo tanto, la empresa aseguradora será la entidad encargada de indemnizar, devolver todo o parte del daño producido según condiciones del contrato. Pero la empresa aseguradora no solo actúa en el momento del siniestro, también asesora y mantiene informado al cliente sobre el nivel de riesgo (Akin, 2009).

El comercio del riesgo puede generar ganancias mutuas, es decir las ganancias que se generan cuando la gente menos dispuesta a afrontar el riesgo se lo transfiere a personas con mayor disposición a soportarlo. Y que parte del riesgo se puede eliminar por la diversificación.

En el principio de diversificación existe un agente importante que se denomina Reaseguro. Las empresas reaseguradoras son también empresas de seguros que asumen el riesgo de otra empresa aseguradora. Es un acuerdo mediante el cual un asegurador, denominado cedente, transfiere a otro 
asegurador, denominado reasegurador, la totalidad o una parte de sus riesgos y éste recibe una parte de la prima. Este mecanismo permite estabilidad para las compañías de seguros así como tranquilidad para los asegurados sobre posibles riesgos que de que la empresa aseguradora no pueda cubrir la indemnización.

Existen tipos de compañías de seguros como compañías de seguros mutuos y sociedades por acciones, la diferencia radica en que la primera utiliza solo el capital acumulado de los clientes, mientras que la otra tiene una entrega de capital inicial por parte de los accionistas. El capital es invertido para el propósito de ganancia.

Las entidades aseguradoras se encuentran expuestas, además de los riesgos generales a que se expone toda entidad financiera (como son, entre otros, los riesgos de crédito, mercado, liquidez, operacional, legal, estratégico y reputación), a riesgos particulares de la actividad aseguradora como serían los riesgos de suscripción y de insuficiencia de reservas técnicas. Estos últimos también suponen la posibilidad de afectar los resultados potenciales y, por ende, de generar una pérdida del valor económico del patrimonio de las entidades aseguradoras y de su reputación en el mercado con efectos nocivos para su solvencia y viabilidad financiera.

\section{Los seguros en el Ecuador}

El seguro en el Ecuador tuvo origen en la época republicana, surgió como una necesidad eminentemente social y comercial para generar beneficios económicos y la protección de bienes de las personas que lo contratan y pagan un valor por dicho servicio. Alvear (2009) en su obra "Introducción al Derecho de Seguros" menciona los orígenes de la Institución del Seguro en el Ecuador y señala que ésta surgió de manera rudimentaria en la época republicana, puesto que ciertos agentes tenían la labor de ser los receptores y mensajeros de Compañías Extranjeras, que en el Ramo de Incendio cubrían ciertas necesidades de la población ecuatoriana.

La actividad aseguradora comenzó a funcionar con regulación jurídica a partir del año 1909, mientras que recién en el año de 1933 se expidió una ley que concedía a la Superintendencia de Bancos cierto control sobre la actividad de seguros y señalaba que "las compañías de seguros deben cooperar al desarrollo de la economía nacional, mediante inversiones en el país de sus capitales y reservas”. 
En el Ecuador aparece el seguro con agentes de compañías extranjeras cuya misión era de receptores y mensajeros. Durante el siglo XIX, la actividad aseguradora era libre e independiente del Estado. Los aseguradores que trabajaban en su propio país operaban en el puerto de Guayaquil en los ramos de incendio y marítimo.

El primer indicio que se tiene es en el Código de Comercio de Eloy Alfaro, publicado por Decreto el 30 de Julio de 1906, en el cual se hace una pequeña mención entre los actos de Comercio al Contrato de Seguro (Icaza, 1995).

Claramente las empresas extranjeras eran las únicas participantes. E1 21 de octubre de 1909 se emite un decreto para reglamentar el funcionamiento de las compañías en el país, se establecieron normas como la presencia de un apoderado, un capital mínimo para operar, inversión del 25\% declarado en bienes raíces, cédulas hipotecarias y otros papeles fiduciarios (Icaza, 2005).

En Ecuador a finales del Siglo XIX existían sólo empresas fundadas en el extranjero que ofrecían pólizas de seguros para Transporte e Incendio, además en 1.933 se le otorga a la Superintendencia de Bancos la función Organismo de Control, en el año 1940 se crea la primera compañía de seguros, La Nacional Compañías de Seguros Generales S.A., y en 1.943 se crea La Unión Compañía Nacional de Seguros S.A. De esta forma se dan los cimientos para la evolución de las coberturas de las pólizas de seguros en Ecuador. En 1943 se crea la Póliza de Aviación. En 1945 se incorpora el Seguro de Fianzas de Fidelidad. En 1946 se implementa el Ramo de Automotores. En 1947 se introduce el Ramo de Accidentes Personales y Robo con Fractura.

En 1950 se implanta la Póliza de Mortalidad de Ganado y Seguros de Accidentes de Trabajo. En 1953 se establece el Seguro de Garantías y Cumplimientos de Contratos. En 1956 se ofrece la Póliza de Vida Individual conocida como "Póliza Económica". En 2006 se introduce la Póliza de Exequias. En 2007 se presenta al mercado la póliza de seguros de Premios y Seguros Odontológicos. En 2008 se oferta al mercado el Seguro Integral para Pymes. En la actualidad el sistema asegurador ecuatoriano está integrado por 38 compañías de seguros legalmente establecidas, 30 compañías atienden el segmento de mercado de Seguros General y 8 operan con el Ramo de Vida.

A inicios del siglo XX, los únicos ramos que operaban son: Seguros de Vida, Incendio y Marítimo. El 31 de diciembre de 1937, el Estado dicta la ley sobre Inspección y Control de Seguros que 
complementa la regulación a las empresas. Con la ley se construye un control administrativo, financiero y económico sobre la actividad aseguradora en el Ecuador. A partir de 1937 se crean compañías nacionales y se empieza a motivar a inversionistas nacionales y al Estado de promocionar empresas de seguros con capital nacional, así empiezan a formarse compañías nacionales (Icaza, 2005).

En la década de 1950 a 1960 operaban compañías en los ramos de robo, fidelidad, responsabilidad civil y vehículos. En 1963 se reforma la Ley de Seguros mediante Decreto Supremo de la Junta Militar de Gobierno y en 1967 se dicta la Ley General de Compañías de Seguro que establece los requisitos que debe cumplir una compañía para operar seguros de vida y daños en el país. También se regula a los corredores de seguro, agencias colocadoras de seguro, etc. Y además se nombra a la Superintendencia de Bancos ser la institución reguladora de las compañías de seguros. A partir de esta fecha, la Superintendencia de Bancos del Ecuador reguló y controló a las empresas de seguros y a la vez la dictó regulaciones y parámetros bajo los cuales debía regirse la actividad aseguradora. (Icaza, 2005).

En el Ecuador, actualmente el seguro desempeña un papel fundamental en la economía, debido a su contribución al desarrollo económico, lo que origina nuevas exigencias al sector. Esta contribución se ve reflejada a través de su aporte al PIB, en la generación de empleos en compañías de seguros, intermediarios, agencias productoras de seguros y mediante la protección de los individuos y sus bienes frente a las consecuencias de los riesgos, transformando los costos impredecibles de una pérdida en costos fijos asumibles a través de las primas.

El mercado asegurador ecuatoriano ha experimentado importantes cambios y transformaciones, especialmente por reformas orientadas a regularlo, empezando por la supervisión y control del mercado que cambia de la Superintendencia de Bancos a la Superintendencia de Compañías, Valores y Seguros.

\section{A manera de conclusión, reflexiones finales}

Luego de haber realizado la extensa revisión del discurso de los diferentes autores consultados para el presente artículo, se quiere terminar no con un cuerpo de conclusiones, sino más bien con unas reflexiones. Queda claro que las compañías de seguros tienen como objetivo brindar protección a las 
personas y empresas frente a la ocurrencia de un riesgo. El seguro forma parte de las acciones que puede tomar un individuo para una adecuada gestión de riesgos. La transferencia de los mismos se realiza a cambio del pago de una cantidad de dinero a la compañía aseguradora.

Las compañías aseguradoras contribuyen con la economía al proteger a los individuos y empresas de pérdidas financieras, y mediante la inversión del dinero que recauda de las primas pagadas por los asegurados para expandir sus operaciones.

El desarrollo del mercado asegurador depende del comportamiento económico del país y se refleja en dos indicadores: el aumento de la participación de las primas, y la del mercado asegurador. Como se ha evidenciado en las revisiones respectivas, el mercado asegurador ecuatoriano es una industria que muestra crecimiento y puede llegar a tener mayores niveles de participación en la economía porque la tasa de crecimiento del mercado es mayor a la tasa de crecimiento del Producto Interno Bruto del Ecuador en los últimos años, ya que se evidencio que el crecimiento del sector tuvo una correlación positiva con la coyuntura macroeconómica del país reflejando que sí es afectado por el desenvolvimiento de la economía nacional.

Por otro lado, hay que destacar que el mercado asegurador ecuatoriano no es un mercado consolidado debido a que existen empresas con capitales variados. Las empresas grandes poseen mayor aceptación en el mercado y abarcan la mayoría de clientes. Además, la demanda en seguros del mercado ecuatoriano es baja en relación al número de empresas oferentes. Esto produce un mercado con poca solidez, y además afectan los precios de las pólizas.

Por ello se puede decir que el mercado asegurador ecuatoriano es un mercado débil porque está conformado por empresas con poca autonomía ya que no tienen la capacidad de asumir sus riesgos directamente, sino sus pólizas son administradas por reaseguradoras.

Las reaseguradoras son importantes para ayudar a distribuir riesgos, aumentar la capacidad de la aseguradora de asumir mayores riesgos a los que normalmente podría aceptar, equilibrar las carteras de las empresas de seguros, y a la vez tener un respaldo y mayor confianza. Es necesario que las pólizas se agrupen por tipos para obtener carteras homogéneas, de esa manera, mientras más primas de un solo tipo existan, la probabilidad de ocurrencia del riesgo es menor y los costos por siniestros se compensan. Sin embargo, en Ecuador las cifras de reaseguro sobrepasan más de la mitad de la 
cartera indicando un alto nivel de emisión de pólizas heterogéneas y que las empresas aseguradoras no tienen la capacidad para administrar los riesgos.

En cuanto a la eficiencia de costos y gastos, las empresas aseguradoras en el Ecuador tienen altos gastos administrativos en relación a la prima retenida neta, inclusive teniendo más de la mitad de riesgos cedidos a reaseguradoras. Esto lo convierte en un mercado ineficiente en términos de gastos y por consiguiente disminuye sus ganancias y utilidad técnica, pero como ya se ha dicho, el mercado asegurador ecuatoriano no es un mercado concentrado. Sin embargo, por separado tiene ramos altamente concentrados que son Salud y Accidentes en donde una empresa abarca la mayor parte del sector. Esto es una desventaja debido a que la falta de competencia afecta los precios y la calidad del servicio, no existe motivación de mejorar estándares de publicidad, calidad, tecnología, conocimiento, entre otros, que son factores que ayudan a mejorar la eficiencia del mercado.

Es importante desarrollar la educación en seguros (tener conciencia de la necesidad de asegurarse ante la probabilidad de un siniestro) dentro de la sociedad, en ese aspecto aún existe indiferencia por parte de las personas a contratar seguros por cuenta propia ya sea por precios o porque no creen necesario. Así como se ha desarrollado la cultura del seguro en autos, se debería dar mayor educación a los usuarios sobre los demás ramos.

Es necesario mejorar los niveles de supervisión de los ramos con mayor cesión y de aquellas empresas que más cedan riesgos, de esta forma mejorar el control de las carteras de estas empresas y las respectivas autorizaciones para operar en los ramos, para así determinar si es conveniente que la empresa mantenga la autorización y esté en condiciones de asumir los riesgos. Tratar de poner límites en los porcentajes de cesión de acuerdo a los ramos, probablemente, existirán algunos que no son necesarios ceder y que las aseguradoras puedan asumir directamente. Con la finalidad de disminuir el porcentaje de cesión de primas que no se había controlado anteriormente.

Se debe mejorar el análisis de riesgos de inversiones en el mercado ecuatoriano, de esta forma quedarse con aquellas que tengan la mejor relación entre riesgo y rendimiento, y así mejorar la calidad del activo y a la vez tener el rendimiento que le permita pagar obligaciones a corto plazo, ya que son rubros altos en las aseguradoras ecuatorianas. 
En Ecuador se debe propender a una estabilidad jurídica con el fin de que las empresas aseguradoras tengan claras las reglas del juego y estas no sean modificadas con frecuencia para poder tomar decisiones a mediano y largo plazo. Además de atraer nuevas inversiones en este sector.

\section{Bibliografía.}

Albarrán, L. y Del Pozo, G. (2011) Investigaciones en seguros y gestión de riesgos Editorial: Fundación Mapfre (Instituto de Ciencias del Seguro)

Alvear, J. (2009) Introducción al Derecho de Seguros, Editorial EDINO, Guayaquil- Ecuador.

Becerra, R. (2013). Manual de principios jurídicos del seguro. Texas: Temis Librería.

Picazo, A. (2015). La eficiencia en los seguros. Ecuador; Revista de Economía Aplicada,

Fernández, A. (2008). El Seguro, su estructura y función económicas, Buenos Aires - Argentina. Editorial Nuevo mundo

Durvan S.A. (2010). Gran Especialidad del Mundo. Editorial Marín. Tomo 15. Prestamos de la Gruesa. Primera edición. Bilbao

Akin, B. (2009). What attorneys need to know about Insurance. Tenesse Bar Journal, Arrow. (1964). The Role of Securities in the Optimal Allocation of Risk-bearing.

Arrow, K. (2003). Uncertainty and the Welfare Economics of Medical Care.

Arrow, K. (1971). Essays in the theory of Risk Bearing. Amsterdam.

Avila, M. (2008). Economia. México: Umbral.

Blanco, L. (2000). El Costo del Seguro Privado en el Ecuador.

Borch, K. (2004). The Economic Theory of Insurance. Bergen .

Langlois, R. (2010). Empresas, mercado y cambio económico: Una teoría dinámica de las instituciones empresariales. Madrid: Ediciones 2000.

Ley de seguros y reaseguros del Ecuador. (2014). Quito: Asamblea Nacional del Ecuador. 
Medrano, L. (2008). El papel de las agencias de seguros en la competencia entre compañias aseguradoras. (Spanish). The Role of Agency Insurers in the Competition between Insurance Companies. (English). 301-316.

Molina, G., Vargas J., Berrio, A., y Muñoz, P. (2010). Características de la contratación entre aseguradores y prestadores de servicios de salud, Medellín, 2007-2008. (Spanish). Characteristics of the Contracting out Process between Insurers and Hospitals. 103-115.

Pinto, S. (2001). El Seguro. Editorial Jurídica de Chile.

Tortella, G. (2011). Sobre Los Orígenes Del Seguro En España. Sevilla: Universidad de Alcalá de Henares.

Willet, A. (2001). The Economic Theory of Risk and Insurance 